\title{
Differing Spontaneous Brain Activity in Healthy Adults with Two Different Body Constitutions: A Resting-State Functional Magnetic Resonance Imaging Study
}

\author{
Ching-Hsiung Liu ${ }^{1,2,3} \oplus$, Yen-Ying Kung 2,4 , Tzu-Chen Yeh ${ }^{3,5,8}$, Pei-Shan Hsu ${ }^{3,5,6}$, \\ Ching-Ju Yang 3,5, Chou-Ming Cheng ${ }^{3}$, Hong-Chun Lin ${ }^{4}$, Jen-Lin Yang 4,7, Ta-Peng Wu ${ }^{4}$, \\ Ching-Mao Chang ${ }^{4,7}$, Jen-Chuen Hsieh ${ }^{3,5}$ and Fang-Pey Chen ${ }^{2,4, *}$ \\ 1 Department of Neurology, Lotung Poh-Ai Hospital, Ilan 26514, Taiwan \\ 2 Institute of Traditional Medicine, School of Medicine, National Yang-Ming University, Taipei 11221, Taiwan \\ 3 Integrated Brain Research Unit, Department of Medical Research, Taipei Veterans General Hospital, \\ Taipei 11267, Taiwan \\ 4 Center for Traditional Medicine, Taipei Veterans General Hospital, Taipei 11267, Taiwan \\ 5 Institute of Brain Science, School of Medicine, National Yang-Ming University, Taipei 11221, Taiwan \\ 6 Department of Chinese Medicine, Taipei Tzu Chi Hospital, Buddhist Tzu Chi Medical Foundation, \\ New Taipei City 23142, Taiwan \\ 7 Faculty of Medicine, School of Medicine, Yang-Ming University, Taipei 11221, Taiwan \\ 8 Department of Radiology, Taipei Veterans General Hospital, Taipei 11267, Taiwan \\ * Correspondence: fpchen@vghtpe.gov.tw; Tel.: +886-2-2875-7454; Fax: +886-2-2875-7452
}

Received: 11 May 2019; Accepted: 28 June 2019; Published: 30 June 2019

\begin{abstract}
Traditional Chinese medicine (TCM) practitioners assess body constitution (BC) as a treatment basis for maintaining body homeostasis. We investigated patterns in spontaneous brain activity in different $\mathrm{BC}$ groups using resting-state functional magnetic resonance imaging (rsfMRI) and determined the relationship between these patterns and quality of life (QOL). Thirty-two healthy individuals divided into two groups (body constitution questionnaire (BCQ)-gentleness [BCQ-G] and BCQ-deficiency [BCQ-D]) based on the body constitution questionnaire (BCQ) underwent rsfMRI to analyze regional homogeneity (ReHo) and the amplitude of low-frequency fluctuation (ALFF). The World Health Organization Quality of Life Instruments (brief edition) scale was used to evaluate the QOL. The BCQ-G group $(n=18)$ had significantly greater ReHo values in the right postcentral gyrus and lower ALFF values in the brainstem than the BCQ-D group $(n=14)$. In the BCQ-D group, decreased ReHo of the postcentral gyrus correlated with better physiological functioning; increased ALFF in the brainstem correlated with poor QOL. BCQ-subgroup analysis revealed a nonsignificant correlation between ReHo and Yang deficiency/phlegm and stasis (Phl \& STA). Nonetheless, the BCQ-D group showed a positive correlation between ALFF and Phl \& STA in the parahippocampus. This study identified differences between BCQ-G and BCQ-D types of healthy adults based on the rsfMRI analysis. The different BCQ types with varied brain endophenotypes may elucidate individualized TCM treatment strategies.
\end{abstract}

Keywords: body constitution; magnetic resonance imaging; quality of life; traditional Chinese medicine

\section{Introduction}

Traditional Chinese medicine (TCM) has been practiced in China for 2000 years. TCM practitioners assess individual innate characters as patterns of body constitution (BC) according to the yin-yang 
theory and treat patients with herbal medicine, tuina, or acupuncture in clinical practice. TCM treats the mind-body as a holistic system [1-3]. Based on TCM theory, diseases originate from an unbalance of yin and yang in the mind-body and the attenuation of $\mathrm{BC}[3,4]$.

The most widely used instruments for BC classification were developed by Wang and $\mathrm{Su}$. Wang et al. established a constitutional rule of nine that forms the primary content of the 'Constitution in Chinese Medicine Questionnaire' (CCMQ). This includes gentleness, qi-deficiency, yang-deficiency, yin-deficiency, phlegm-dampness, dampness-heat, blood-stasis, qi-depression, and special diathesis [5,6]. Su et al. [3,4] created a 42-item questionnaire for assessing BC (body constitution questionnaire [BCQ]), which includes healthy/sub-healthy conditions consisting of gentleness, yang-deficiency (YaD), yin-deficiency (YinD), and phlegm and stasis (Phl \& STA). YaD is characterized by cold intolerance, which may correlate with dysfunction of the hypothalamic-pituitary-adrenal axis [7]; YinD is characterized by heat intolerance, which may correlate with lipid metabolic gene regulation $[7,8]$. Phl \& STA is associated with blood lipid and sugar metabolism [9]. In TCM practice, an individual's BC represents the constitutional traits of an individual's morphosis, including physical and psychological components that determine susceptibility to pathogenic factors $[1,10]$.

Functional magnetic resonance imaging (fMRI) is a useful tool that can link the brain (endophenotype) and behavior (phenotype), including physiological phenomena or pathological disorders of the central nervous system [11,12]. This imaging approach is based on assessing blood oxygen level-dependent (BOLD) signal changes as markers of brain activity changes. Two methods can be used to measure different aspects of brain-behavior interactions: Task-based or resting-state fMRI (rsfMRI) [13,14]. Recently, rsfMRI studies have enabled further understanding of human brain plasticity $[15,16]$. Recent rsfMRI studies have assessed the neural mechanisms of TCM, such as acupuncture treatment, in various diseases $[17,18]$. However, few fMRI studies have assessed the TCM theory $[19,20]$. Differential resting-state brain activity has been detected in patients with yin and yang-type depression [20]. Therefore, neural activity measures may be suited for tracing the early brain function properties of different BCQ groups in healthy adults.

Regional homogeneity (ReHo) and amplitude of low-frequency fluctuation (ALFF) analyses are two vital methods for measuring local neural activity in the brain [21,22]. ReHo analysis is calculated from the direct neighborhood of single voxels for measuring the time-series similarity of its nearest neighbors [23]. ALFF estimates the total power of the BOLD signal within a low-frequency range (0.01-0.08 Hz) [22,24]. Previous studies have indicated that ReHo and ALFF show alternations with similar distributions in different pathological conditions $[25,26]$. However, ReHo may be more sensitive than ALFF at detecting regional abnormalities, and ALFF may be more effective at measuring global spontaneous activity [27]. Consequently, the combination of the two analyses may provide more information on regional brain spontaneous activity than either alone [22,27].

In the present study, we used ReHo and ALFF analyses to inspect global spontaneous neuronal activity in the brain of healthy adults with different BCQ types. We hypothesized that different BCQ types of healthy adults would have different ReHo and ALFF maps related to brain regions. Further, changes of the spontaneous brain activity would be related to the behavior scales and the sub-patterns of BCQ-related measurements.

\section{Methods}

\subsection{Study Design and Participants}

This study was approved by the institutional review board of the Taipei Veterans General Hospital. All participants provided written informed consent. We recruited 35 healthy individuals from the local community via advertisements. Inclusion criteria were: Age 35-70 years; no organic brain disorder; no current use of medications for chronic illness; and no history of any psychiatric/neurological disease or surgery. All participants completed the BCQ and World Health Organization Quality of Life Instruments (brief edition) (WHOQOL-BREF) questionnaires before undergoing rsfMRI. 
The WHOQOL-BREF has four subdomains: (1) Physical domain (score range, 4-20); (2) psychological domain (score range, 4-20); (3) social domain (score range, 4-20); and (4) environmental domain (score range, 4-20). The total score ranges 8-80 points, with higher values indicating better QOL [28].

Three individuals were excluded from this study because of claustrophobia during the MRI examination $(n=1)$ or organic brain lesions, revealed by MRI $(n=2)$. Thus, finally, we included 32 healthy individuals and divided them into two groups based on the BCQ. BCQ-gentleness (BCQ-G) type was defined as individuals with no YaD, YinD, or Phl \& STA. BCQ-deficiency (BCQ-D) type was defined as individuals with YaD or YinD. The BCQ contains three subtypes: YaD (score range, 19-95), YinD (score range, 19-95), and Phl \& STA (score range, 16-80); the cut-off points for the scores of YinD, $\mathrm{YaD}$, and Phl \& STA are $\geq 30$ points, 31 points, and 27 points, respectively.

A flowchart of the study design is shown in Supplementary Figure S1.

\subsection{MRI Acquisition}

fMRI was performed with a 3.0 Tesla scanner (Discovery MR750, GE Healthcare, Waukesha, WI, USA) at the Integrated Brain Research Unit, Department of Medical Research \& Education, Taipei Veterans General Hospital, Taipei, Taiwan. A 12-channel head coil was used, along with restraining vacuum pads to minimize head motion and to reduce scanner noise. Sequence of gradient echo-planar image (EPI) was used for the rsfMRI scan with the following parameters: Repetition number $=200$ for conventional EPI; dummy scan, 5; TR (repetition time) $/ \mathrm{TE}\left(\right.$ echo time) $=2000 / 30 \mathrm{~ms}$; flip angle $=90^{\circ}$; matrix size $=64 \times 64 \times 40$; slice number $=40$; slice thickness $=3 \mathrm{~mm}$; and field of view $(F O V)=240 \times 240 \mathrm{~mm}^{2}$.

The baseline resting brain activity was acquired as follows: (1) The first five EPI scans of each rsfMRI series were discarded for signal saturation and magnetic-field stabilization; and (2) during the 7-min fMRI scanning, individuals were instructed to keep their eyes closed, relax, move as little as possible, and stay awake, for which they were asked to hold a plastic ball. An online real-time analysis of head motion using the methods modified from Analysis of Functional Neuroimaging (NIMH, Bethesda, MD, USA) was performed to confirm the quality of rsfMRI images, with a head translation of $1 \mathrm{~mm}$ and head rotation $<0.5^{\circ}$ for each session. If the head motion exceeded the motion criteria, data were excluded from the analysis. Structural images were acquired using a three-dimensional T1-weighted structural image $\left(\mathrm{TR} / \mathrm{TE} / \mathrm{TI}\right.$ [inversion time] $=8.2 / 3.2 / 450 \mathrm{~ms}$; flip angle $=12^{\circ}$; matrix size $=256 \times 256 \times 176 ;$ FOV $=230 \times 230 \mathrm{~mm}^{2} ;$ and voxel size $=0.9 \times 0.9 \times 0.9 \mathrm{~mm}^{3}$ ). The total rsfMRI scan time was approximately $11 \mathrm{~min}$. Head cushions and earplugs were provided to reduce head motion and noise during the scans.

\subsection{Data Preprocessing}

fMRI data preprocessing was performed using the toolbox Data Processing Assistant for Resting-state fMRI V4.4 advanced edition (DPARSF-A, Key Laboratory of Behavioral Science and Magnetic Resonance Imaging Research Center, Institute of Psychology, Chinese Academy of Sciences) [29] with Statistical Parametrical Mapping 12 (SPM; https://www.fil.ion.ucl.ac.uk/spm/ software/spm12) in MATLAB 2014a (The Math Works, Inc., Natick, MA, USA). Functional image data were corrected for any head movements using SPM-realign, a linear transformation procedure. We coregistered structural data with SPM-coregister and normalized to Montreal Neurological Institute (MNI) space using a standard MNI template (SPM-normalize). Next, the data were smoothed with SPM-smooth. During the fMRI examination, the participant's vital signs, such as heart rate, oxygen saturation, and respiratory rate, were monitored and recorded.

\subsection{ALFF and ReHo Analyses}

ALFF and ReHo analyses were performed based on previous studies [23,24] using the Data Processing \& Analysis for Resting-State Brain Imaging (DPABI) [29]. For ALFF analysis, the resampled functional images were smoothed with a 3D Gaussian kernel of $4 \mathrm{~mm}$ full-width at half-maximum (FWHM). De-trend and band-pass filtering $(0.01-0.08 \mathrm{~Hz})$ were performed to remove the effects of 
low-frequency drift and high-frequency noise. The following nuisance variables were regressed out: (1) Six head movement parameters computed based on rigid body translation SPM12; (2) mean signal within the lateral ventricles of cerebrospinal fluid; and (3) mean signal within a deep white matter region (centrum ovale). Subsequently, the time series were transformed into the frequency domain using a Fourier transform. Next, the square root of the power spectrum was calculated and averaged across $0.01-0.08 \mathrm{~Hz}$ within each voxel to obtain a raw ALFF map. Following this, the global mean ALFF value was calculated by extracting and averaging the raw values from all voxels across the whole brain. Finally, ALFF values for each voxel were divided by the global mean ALFF value for standardization. The resulting ALFF value in a given voxel reflected the degree of its raw ALFF value relative to the average ALFF value of the whole brain [24].

ReHo analysis was performed on the functional images after preprocessing. The nuisance variables were regressed out using the same methods for calculating the ALFF value. Briefly, after de-trend and band-pass filtering were performed, ReHo maps were produced by calculating the concordance of the Kendall coefficient of the time series of a given voxel with its 26 nearest neighbors [23]. Next, the ReHo value of each voxel was standardized by dividing the raw value by the global mean ReHo value. Finally, ReHo data were smoothed using a 3D Gaussian kernel of $4 \mathrm{~mm}$ FWHM for further statistical analysis.

\subsection{Statistical Analysis}

Demographic and behavioral data were compared with the $\chi^{2}$ test (categorical variables) and independent $t$-test (continuous variables) using SPSS (Version 19.0, SPSS Inc., Chicago, IL, USA). Pearson's correlation analysis was applied to assess the degree of association between two variables. Results were considered statistically significant if $p<0.05$.

\subsubsection{Within-Group Analysis}

One-sample $t$-tests were performed on the ALFF and ReHo maps for each group to display the most significant results and reflect the intrinsic characters of these two groups. A conservative statistical significance was set at voxel level $p<0.001$ and cluster size $>150$ voxels, which corresponded to a multiple comparisons correction with family-wise error rate (FWE) of $p<0.05$.

\subsubsection{Between-Group Analysis}

Independent two-sample $t$-tests were performed with a gray matter mask to investigate the between-group differences of ALFF and ReHo values. Global signal regression was imported, and age and sex were included as covariates. The statistical voxel threshold was set at $p<0.005$ and a cluster size $>35$ voxels, which corresponded to an FWE correction of $p<0.05$.

\subsubsection{Correlation Analysis}

We performed a correlation analysis to investigate the association between ALFF/ReHo values and the behavior scales (WHOQOL-BREF and BCQ). WHOQOL-BREF and its subdomains were used to assess multiple factors of QOL associated with adaptive or maladaptive changes in the brain region. To explore the most significant correlations among the ALFF/ReHo MRI values, the statistical threshold was set at voxel level $p<0.005$ and cluster size $>35$ voxels, which corresponded to a $p<0.05$ (FWE-corrected). 


\section{Results}

\subsection{Demographic Data}

A total of 32 participants were included in the data analysis. The BCQ-G and BCQ-D groups did not differ statistically in terms of age, sex, body mass index, and scores of WHOQOL-BREF. However, there were significant differences in BCQ-subtype between the groups $(p<0.001$; Table 1$)$.

Table 1. Demographic data in different body constitution questionnaire-deficiency (BCQ) groups.

\begin{tabular}{cccc}
\hline Characteristics & BCQ-G $(\boldsymbol{n}=\mathbf{1 8})$ & BCQ-D $(\boldsymbol{n}=\mathbf{1 4})$ & $\boldsymbol{p}$-Value \\
\hline Gender (M/F) & $9 / 9$ & $6 / 8$ & 0.688 \\
Age (years) & $52.0 \pm 6.32$ & $46.36 \pm 9.95$ & 0.396 \\
BMI & $23.68 \pm 2.39$ & $24.04 \pm 4.56$ & 0.794 \\
BCQ Subtype & & & \\
YinD & $25.78 \pm 2.92$ & $34.64 \pm 5.02$ & $0.000 * *$ \\
YaD & $25.28 \pm 2.65$ & $37.21 \pm 4.95$ & $0.000^{* *}$ \\
Phl \& STA & $20.06 \pm 3.03$ & $27.21 \pm 4.84$ & $0.000 *$ \\
WHOQOL-BREF, total & $61.38 \pm 8.28$ & $58.25 \pm 7.24$ & 0.27 \\
Physical & $16.10 \pm 1.82$ & $15.51 \pm 1.52$ & 0.341 \\
Psychological & $14.78 \pm 2.22$ & $13.71 \pm 2.33$ & 0.199 \\
Social & $15.26 \pm 2.30$ & $14.38 \pm 3.10$ & 0.390 \\
Environmental & $15.25 \pm 2.35$ & $14.64 \pm 1.20$ & 0.386 \\
\hline
\end{tabular}

Data are mean \pm SD and were compared using the $\chi^{2}$ test (categorical variables) and independent $t$-test (continuous variables). BCQ-G, body constitution questionnaire-gentleness type; BCQ-D, body constitution questionnaire-deficiency type; BMI, body mass index; YinD, yin-deficiency; YaD, yang-deficiency; Phl \& STA, phlegm and stasis; WHOQOL-BREF, World Health Organization Quality of Life Instruments (brief edition). ${ }^{* *} p<0.01$.

\subsection{Behavior Scale Correlations in the Different Groups}

$\mathrm{YaD}$ and Phl \& STA scores were negatively correlated with several variables, such as the psychological domain and sum of the WHOQOL-BREF scores. However, there was no significant correlation between BCQ subscores and WHOQOL-BREF when the groups were divided into BCQ subtypes (Table 2).

\subsection{ReHo and ALFF Analyses}

In the BCQ-G group, both ReHo and ALFF signals were significantly higher than the global mean values in the posterior cingulate cortex (PCC). In addition, no region showed a lower signal than the average values in both analyses. In the BCQ-D group, the orbitofrontal cortex (OFC) showed higher ReHo and ALFF values than the global mean values. Further, there were higher ReHo values than the global average values in the PCC. Interestingly, the BCQ-D group revealed lower ALFF values than the global mean value in the precentral gyrus (Table 3 and Figure 1). Finally, BCQ-G group values were significantly increased in the postcentral gyrus (ReHo) and decreased in the left brainstem/cerebellum (ALFF) when compared with the BCG-D group (Table 3 and Figure 2A,B). 
Table 2. Pearson correlation matrix for the scales between WHOQOL-BREF and BCQ subtypes in different groups

\begin{tabular}{|c|c|c|c|c|c|c|c|c|c|c|c|c|c|c|c|c|}
\hline & & \multicolumn{5}{|c|}{ All Healthy Adult Subjects $(n=32)$} & \multicolumn{5}{|c|}{ BCQ-Gentleness $(n=18)$} & \multicolumn{5}{|c|}{ BCQ-Deficiency $(n=14)$} \\
\hline & & \multicolumn{5}{|c|}{ WHOQOL-BREF (Subdomains) } & \multicolumn{5}{|c|}{ WHOQOL-BREF (Subdomains) } & \multicolumn{5}{|c|}{ WHOQOL-BREF (Subdomains) } \\
\hline & & Phy & Psy & Soc & Evn & Sum & Phy & Psy & Soc & Evn & Sum & Phy & Psy & Soc & Evn & Sum \\
\hline YinD & $\begin{array}{c}\text { Pearson } r \\
p \text { value }\end{array}$ & $\begin{array}{c}-0.226 \\
0.214\end{array}$ & $\begin{array}{c}-0.345 \\
0.53\end{array}$ & $\begin{array}{c}-0.294 \\
0.102\end{array}$ & $\begin{array}{c}-0.256 \\
0.158\end{array}$ & $\begin{array}{c}-0.317 \\
0.077\end{array}$ & $\begin{array}{c}-0.306 \\
0.217\end{array}$ & $\begin{array}{c}-0.395 \\
0.105\end{array}$ & $\begin{array}{c}-0.323 \\
0.191\end{array}$ & $\begin{array}{c}-0.206 \\
0.412\end{array}$ & $\begin{array}{c}-0.333 \\
0.177\end{array}$ & $\begin{array}{c}-0.019 \\
0.949\end{array}$ & $\begin{array}{c}-0.185 \\
0.528\end{array}$ & $\begin{array}{c}-0.244 \\
0.400\end{array}$ & $\begin{array}{c}-0.310 \\
0.280\end{array}$ & $\begin{array}{c}-0.220 \\
0.451\end{array}$ \\
\hline $\mathrm{YaD}$ & $\begin{array}{c}\text { Pearson } r \\
p \text { value }\end{array}$ & $\begin{array}{c}-0.246 \\
0.174\end{array}$ & $\begin{array}{c}-0.430 \text { * } \\
0.014 \text { * }\end{array}$ & $\begin{array}{c}-0.313 \\
0.081\end{array}$ & $\begin{array}{c}-0.277 \\
0.125\end{array}$ & $\begin{array}{c}-0.358 \text { * } \\
0.044^{*}\end{array}$ & $\begin{array}{c}-0.243 \\
0.332\end{array}$ & $\begin{array}{c}-0.465 \\
0.052\end{array}$ & $\begin{array}{c}-0.413 \\
0.089\end{array}$ & $\begin{array}{c}-0.239 \\
0.340\end{array}$ & $\begin{array}{c}-0.375 \\
0.125\end{array}$ & $\begin{array}{c}-0.172 \\
0.556\end{array}$ & $\begin{array}{c}-0.469 \\
0.091\end{array}$ & $\begin{array}{c}-0.316 \\
0.270\end{array}$ & $\begin{array}{c}-0.453 \\
0.104\end{array}$ & $\begin{array}{c}-0.398 \\
0.159\end{array}$ \\
\hline P\&S & $\begin{array}{c}\text { Pearson } r \\
p \text { value }\end{array}$ & $\begin{array}{c}-0.165 \\
0.368\end{array}$ & $\begin{array}{c}-0.412 * \\
0.019 *\end{array}$ & $\begin{array}{c}-0.241 \\
0.183\end{array}$ & $\begin{array}{c}-0.208 \\
0.254\end{array}$ & $\begin{array}{c}-0.293 \\
0.104\end{array}$ & $\begin{array}{l}0.034 \\
0.893\end{array}$ & $\begin{array}{c}-0.294 \\
0.236\end{array}$ & $\begin{array}{c}-0.274 \\
0.271\end{array}$ & $\begin{array}{c}-0.158 \\
0.531\end{array}$ & $\begin{array}{c}-0.202 \\
0.421\end{array}$ & $\begin{array}{c}-0.170 \\
0.561\end{array}$ & $\begin{array}{c}-0.416 \\
0.139\end{array}$ & $\begin{array}{c}-0.125 \\
0.669\end{array}$ & $\begin{array}{c}-0.158 \\
0.589\end{array}$ & $\begin{array}{c}-0.250 \\
0.389\end{array}$ \\
\hline
\end{tabular}

BCQ body constitution questionnaire; YinD, yin-deficiency; YaD, yang-deficiency; P\&S, phlegm and stasis; WHOOOL-BREF, World Health Organization Quality of Life Instruments (brief edition); Phy, physical domain of WHOQOL-BREF; Psy, psychological domain of WHOQOL-BREF; Soc, social domain of WHOQOL-BREF; Evn, environmental domain of WHOQOL-BREF; $* p<0.05$ (2-tailed); $r$, Pearson correlation coefficients. 
Table 3. Peak Montreal Neurological Institute (MNI) coordinates for regions exhibiting significant regional homogeneity (ReHo)/amplitude of low-frequency fluctuation (ALFF) values between the different body constitution groups.

\begin{tabular}{|c|c|c|c|c|c|c|c|}
\hline \multirow{2}{*}{ Contrast } & \multirow{2}{*}{ Region } & \multirow{2}{*}{ BA } & \multirow{2}{*}{ Size } & \multirow{2}{*}{$t$ Score } & \multicolumn{3}{|c|}{ Peak Coordinate } \\
\hline & & & & & $x$ & $\mathbf{y}$ & $\mathbf{z}$ \\
\hline \multicolumn{8}{|l|}{ ReHo } \\
\hline \multicolumn{8}{|l|}{ Within-group ${ }^{* *}$} \\
\hline \multicolumn{8}{|l|}{ BCQ-Gentleness } \\
\hline Gentleness $>0$ & PCC, Left & 23 & 16,838 & 33.85 & -3 & -48 & 24 \\
\hline Gentleness $<0$ & NS & & & & & & \\
\hline \multicolumn{8}{|l|}{ BCQ-Deficiency } \\
\hline \multirow[t]{2}{*}{ Deficiency $>0$} & PCC, Left & 23 & 8516 & 30.36 & -3 & -54 & 30 \\
\hline & OFC, Right & 11 & 4593 & 21.54 & 42 & 52 & -12 \\
\hline Deficiency $<0$ & NS & & & & & & \\
\hline \multicolumn{8}{|l|}{ Between-group * } \\
\hline Gentleness > Deficiency & Postcentral gyrus & 2 & 69 & 5.37 & 51 & -24 & 45 \\
\hline Gentleness $<$ Deficiency & NS & & & & & & \\
\hline \multicolumn{8}{|l|}{ ALFF } \\
\hline \multicolumn{8}{|l|}{ Within-group ** } \\
\hline \multicolumn{8}{|l|}{ BCQ-Gentleness } \\
\hline Gentleness $>0$ & PCC & 23 & 11639 & 17.34 & -3 & -54 & 21 \\
\hline Gentleness $<0$ & NS & & & & & & \\
\hline \multicolumn{8}{|l|}{ BCQ-Deficiency } \\
\hline Deficiency $>0$ & OFC, Left & 11 & 161 & 6.79 & -33 & 33 & -18 \\
\hline Deficiency $<0$ & Precentral gyrus, Left & 6 & 29,955 & 52.63 & -18 & -18 & 54 \\
\hline \multicolumn{8}{|l|}{ Between-group * } \\
\hline Gentleness > Deficiency & NS & & & & & & \\
\hline Gentleness $<$ Deficiency & Pons/Cerebellum, Left & & 85 & -3.86 & -12 & -36 & -54 \\
\hline
\end{tabular}

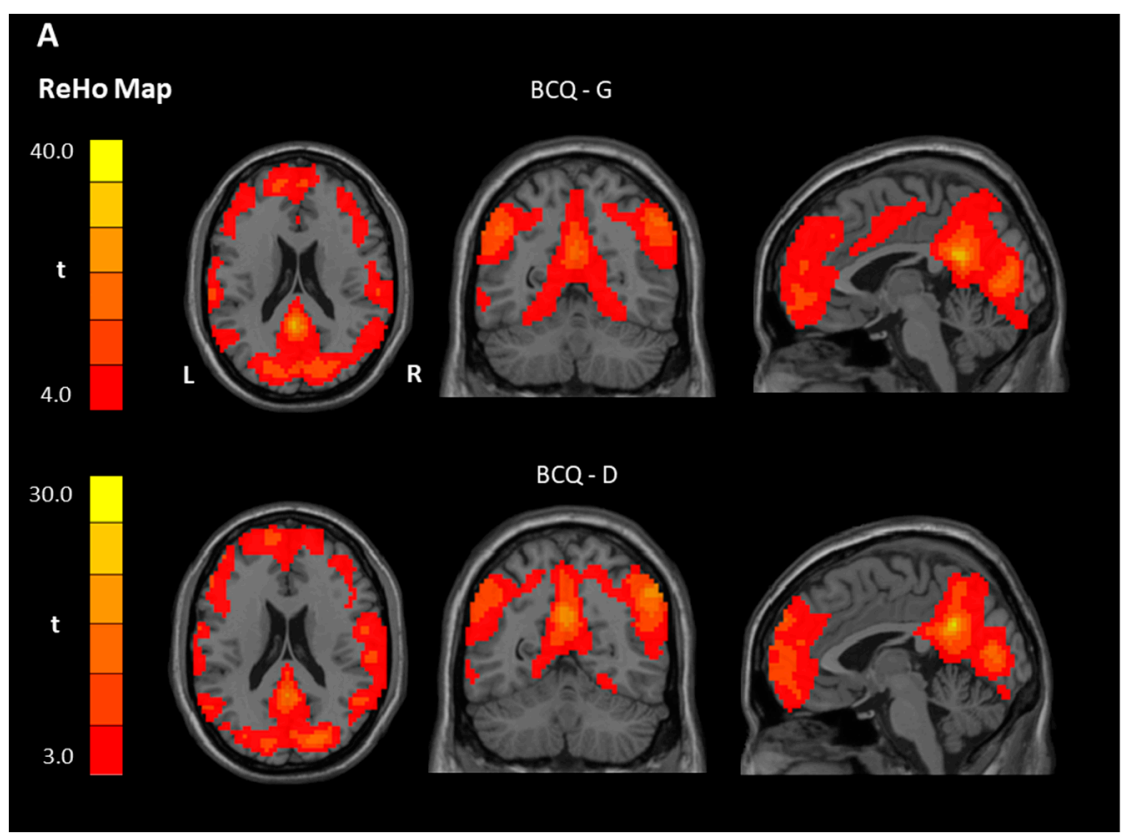

Figure 1. Cont. 


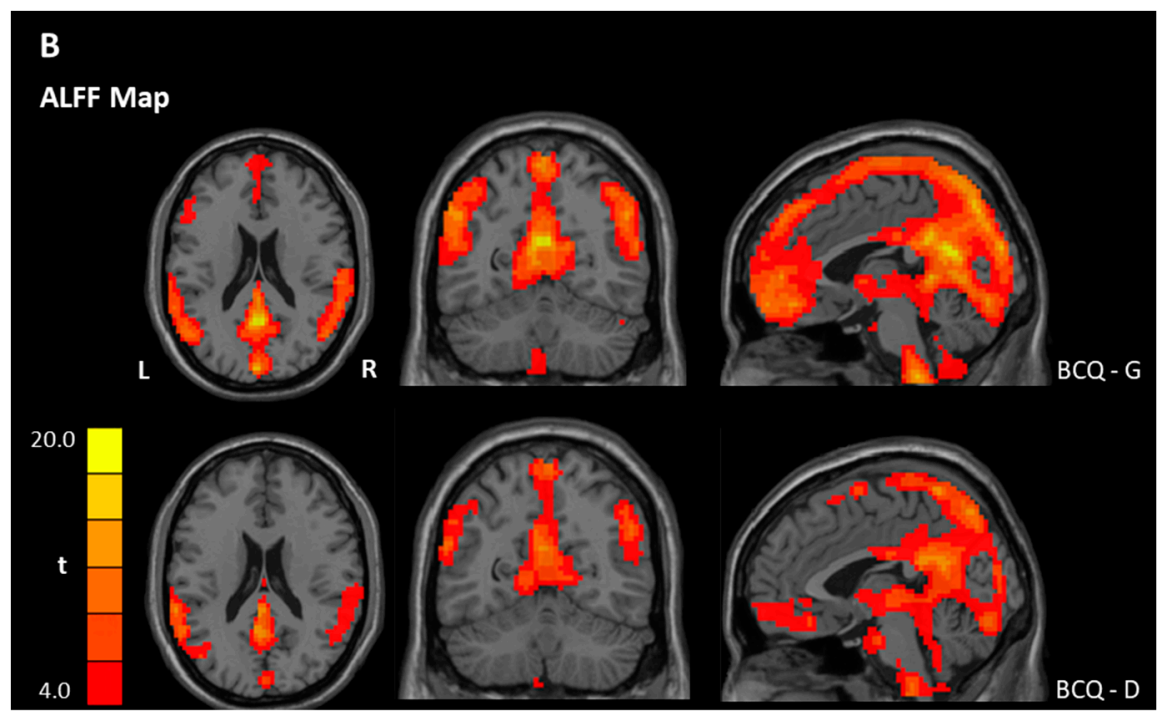

Figure 1. Representative one-sample $t$-test results of ReHo and ALFF maps $(p<0.005$, family-wise error rate (FWE) corrected). Within each group, standardized ReHo (A) and ALFF (B) values in the PCC and the medial PFC were significantly greater than the global mean values in both groups. Other regions, such as the inferior parietal lobe and bilateral occipital lobes, also had greater spontaneous activity. Note that the brain regions were mainly present in the default-mode network, which was not significantly different between the groups in both analyses. ReHo, regional homogeneity; ALFF, amplitude of low-frequency fluctuation; R, right; L, left; BCQ-G, gentleness type of body constitution questionnaire; BCQ-D, deficiency type of body constitution questionnaire; FWE, family-wise error rate; PCC, posterior cingulate cortex; PFC, prefrontal cortex.

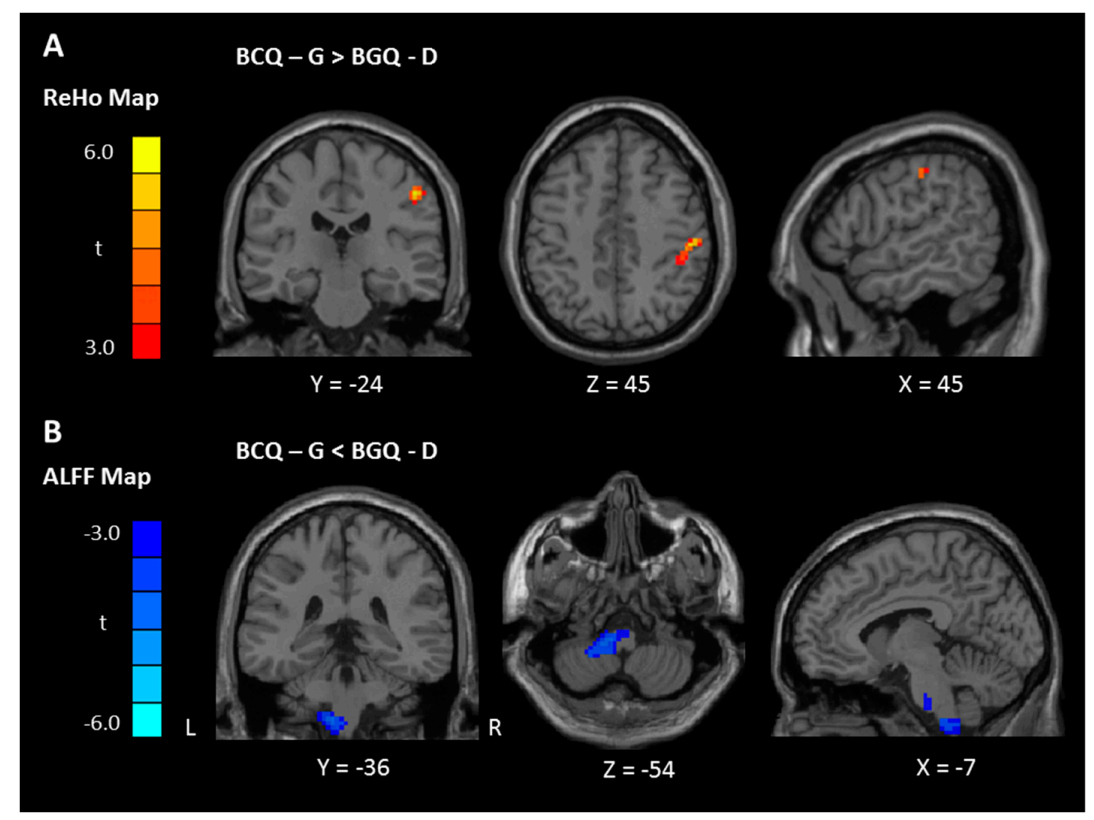

Figure 2. ReHo (A) and ALFF (B) differences between BCQ-G and BCQ-D healthy subjects $(p<0.05$, FWE corrected). A: Compared with BCQ-D subjects, Subjects with significantly increased ReHo in the postcentral gyrus (red). B: Compared with BCQ-D subjects, BCQ-G type showed significantly decreased ALFF in the brainstem (pons) and cerebellum (blue). ReHo, regional homogeneity; ALFF, amplitude of low-frequency fluctuation; BCQ-G, gentleness type of body constitution questionnaire; BCQ-D, deficiency type of body constitution questionnaire; FWE, family-wise error rate; color scale denotes the t value. $x, y, z$, Montreal Neurological Institute coordinates; R, right; L, left. 


\subsection{Correlation Analysis}

Increased ReHo values in the cerebellum/lingual gyrus were positively correlated with the physical subdomains of WHOQOL-BREF in the BCQ-D group when compared with the BCQ-G group (Figure 3A). There were no significant correlations between ReHo/ALFF values and WHOQO-BREF and its subdomains in the BCQ-G group. Besides, in the BCQ-D group, there were significant correlations between increased neural activity (ReHo/ALFF) in the postcentral gyrus, brainstem, and negative association with ventromedial prefrontal cortex ( $\mathrm{vmPFC}$ ) and physical-social performance, as measured by the total WHOQOL-BREF scores and subdomains (Table 4 and Figure 3B-D).

Table 4. ReHo and ALFF values vary with WHOQOL-BREF scores in healthy adults in the BCQ-D group $(n=14)$.

\begin{tabular}{|c|c|c|c|c|c|c|c|}
\hline \multirow{2}{*}{ Values } & \multirow{2}{*}{ Region } & \multirow{2}{*}{ BA } & \multirow{2}{*}{ Size } & \multirow{2}{*}{$t$} & \multicolumn{3}{|c|}{ Peak Coordinate } \\
\hline & & & & & $\mathbf{x}$ & $\mathbf{y}$ & $\mathbf{z}$ \\
\hline \multicolumn{8}{|c|}{ WHOQOL-BREF } \\
\hline \multicolumn{8}{|c|}{$\mathrm{ReHo}$} \\
\hline \multicolumn{8}{|c|}{ Physiological } \\
\hline Positive & Cerebellum/lingual gyrus, $\mathrm{R}$ & 19 & 77 & 6.99 & 12 & -57 & -6 \\
\hline Negative & Supramarginal gyrus, $\mathrm{R}$ & 40 & 68 & 8.58 & 60 & -21 & 33 \\
\hline \multicolumn{8}{|l|}{ ALFF } \\
\hline \multicolumn{8}{|c|}{ WHOQOL-total } \\
\hline Positive & NS & & & & & & \\
\hline Negative & Brainstem, $\mathrm{R}$ & & 67 & 7.84 & 6 & -21 & -24 \\
\hline \multicolumn{8}{|l|}{ Social } \\
\hline Positive & NS & & & & & & \\
\hline Negative & vmPFC, L & 10 & 168 & 5.75 & -15 & 57 & -6 \\
\hline
\end{tabular}

Peak coordinates refer to the MNI space. Correlation analysis: Significance was set at the voxel level $p<0.005$ followed by the family-wise error rate-corrected cluster level $p<0.05$; ReHo, regional homogeneity; ALFF, amplitude of low-frequency fluctuation; WHOQOL-BREF, World Health Organization Quality of Life Instruments (brief edition); BCQ-D, body constitution questionnaire-deficiency type; BA, Brodmann's area; MNI, Montreal Neurological Institute; vmPFC, ventromedial prefrontal cortex; NS, nonsignificant; R, right; L, left.

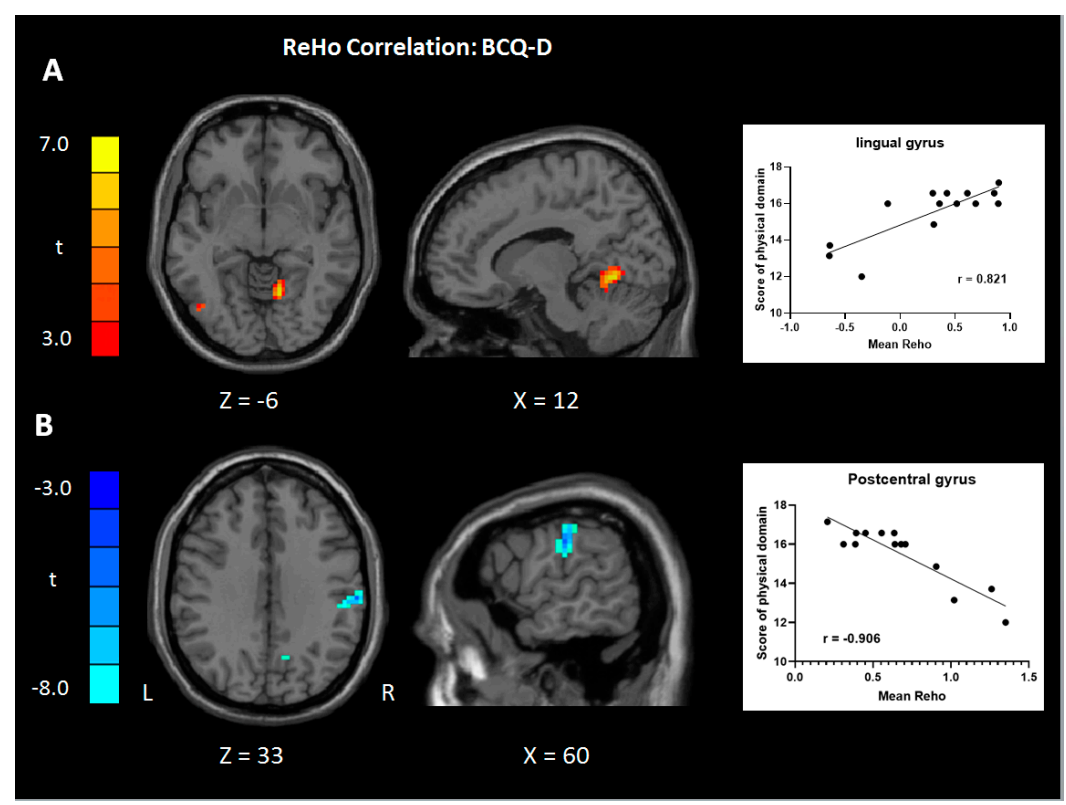

Figure 3. Cont. 


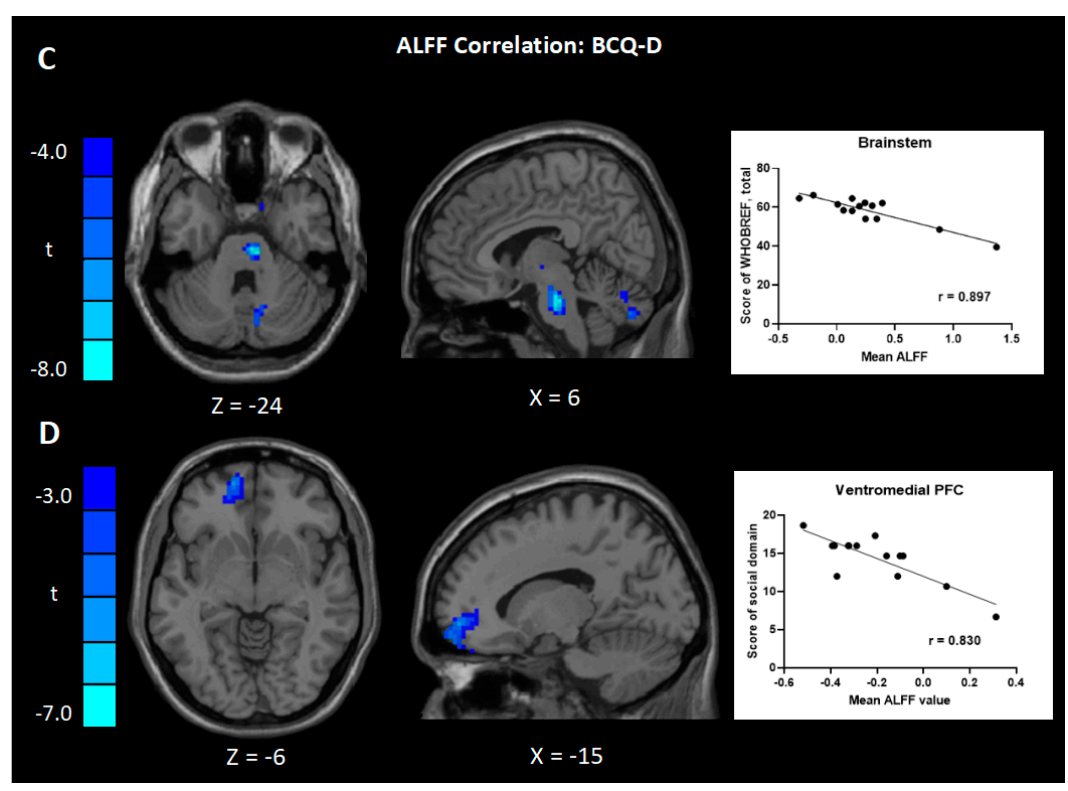

Figure 3. Correlations between the $\operatorname{ReHo}(\mathbf{A}, \mathbf{B})$ and $\operatorname{ALFF}(\mathbf{C}, \mathbf{D})$ values extracted from significantly different regions of BCQ-D subjects ( $p<0.05$, FWE corrected). The mean ReHo values had a significantly positive correlation between the physical domain of WHOQOL-BREF and the region in the right lingual gyrus $(\mathrm{A}, r=0.821, p=0.0003)$, and negative correlation between the physical domain of WHOQOL-BREF and the region in the right posterior central gyrus $(\mathbf{B} ; r=-0.906, p<0.0001)$. The ALFF values had a significantly negative correlation between the total scores of WHOQOL-BREF and the region in the right brainstem $(C ; r=-0.897, p=0.0001)$, and a significantly negative correlation between the social domain of WHOQOL-BREF and the region in the left ventromedial prefrontal cortex. (D; $r=0.830, p=0.0002$ ). ReHo, regional homogeneity; ALFF, amplitude of low-frequency fluctuation; PFC, prefrontal cortex; WHOQOL-BREF, World Health Organization Quality of Life Instruments (brief edition); BCQ-D, deficiency type of body constitution questionnaire; FWE, family-wise error rate; color scale denotes the $t$ value; $x, y, z$, Montreal Neurological Institute coordinates; R, right; L, left.

Next, we pooled all participants into one group to assess the correlation between BCQ subtypes (YinD, Yang D, and Phl \& STA) and ALFF/ReHo values after regressing out the variables of age and sex. There were no significant clusters survived after FWE correction. However, in ALFF analysis, the parahippocampus (Parahippo1, BA 36), OFC, and Parahippo2 (BA 36) were significantly positively correlated with ALFF values in the YaD and Phl \& STA groups (Table 5 and Figure S2).

Table 5. ReHo and ALFF values vary with BCQ subscores in all healthy adults $(n=32)$.

\begin{tabular}{lllllll}
\hline Values & Region & BA & Size & $t$ & \multicolumn{2}{c}{ Peak Coordinate } \\
\cline { 4 - 6 } & & & & & & \\
ReHo & & & & & \\
Yang-deficiency & & & & & \\
Positive & NS & & & & \\
Negative & NS & & & & \\
Yin-deficiency & & & & & \\
Positive & NS & & & & \\
Negative & NS & & & & \\
Phl \& STA & & & & & \\
Positive & NS & & & & \\
Negative & NS & & & & \\
ALFF & & & & & \\
Yang-deficiency & & & & & & \\
\hline
\end{tabular}


Table 5. Cont.

\begin{tabular}{llcccccc}
\hline Values & Region & \multirow{2}{*}{ BA } & Size & $\boldsymbol{t}$ & \multicolumn{3}{c}{ Peak Coordinate } \\
\cline { 4 - 7 } & & & & & $\mathbf{x}$ & $\mathbf{y}$ & $\mathbf{z}$ \\
\hline $\begin{array}{l}\text { Positive } \\
\text { Negative } \\
\text { Yin-deficiency }\end{array}$ & Parahippocampus, L & 36 & 289 & 4.32 & -21 & -18 & -24 \\
$\begin{array}{l}\text { Positive } \\
\text { Negative }\end{array}$ & NS & & & & & & \\
$\begin{array}{l}\text { Phl \& STA } \\
\text { Positive }\end{array}$ & & & & & & & \\
& Parahippocampus, L & 36 & 207 & 5.05 & -24 & -6 & -42 \\
Negative & Orbitofrontal cortex, L & 11 & 304 & 4.52 & -3 & 33 & -15 \\
& NS & & & & & & \\
\hline
\end{tabular}

Peak coordinates refer to the MNI space. Correlation analysis: Significance was set at the voxel level $p$ Peak coordinates refer to the MNI space. Correlation analysis: Significance was set at the voxel level $<0.005$ followed by the family-wise error rate-corrected cluster level $p<0.05$; ReHo, regional homogeneity; ALFF, amplitude of low-frequency fluctuation; BCQ, body constitution questionnaire; Phl \& STA, phlegm and stasis; BA, Brodmann's area; NS, nonsignificant; $R$, right; $L$, left.

Further, we extracted ALFF values from these regions showing significant correlations, which revealed that the BCQ-D group showed increased ALFF values in the Parahippo1, Parahippo2, and OFC regions (Figure 4). In addition, the BCQ-D group has a significant positive correlation between ALFF values and YaD and Phl \& STA scores in the parahippocampus and OFC, respectively (Figure 4; Table 5).

\section{Parahippo1 Orbitofrontal Parahippo 2}

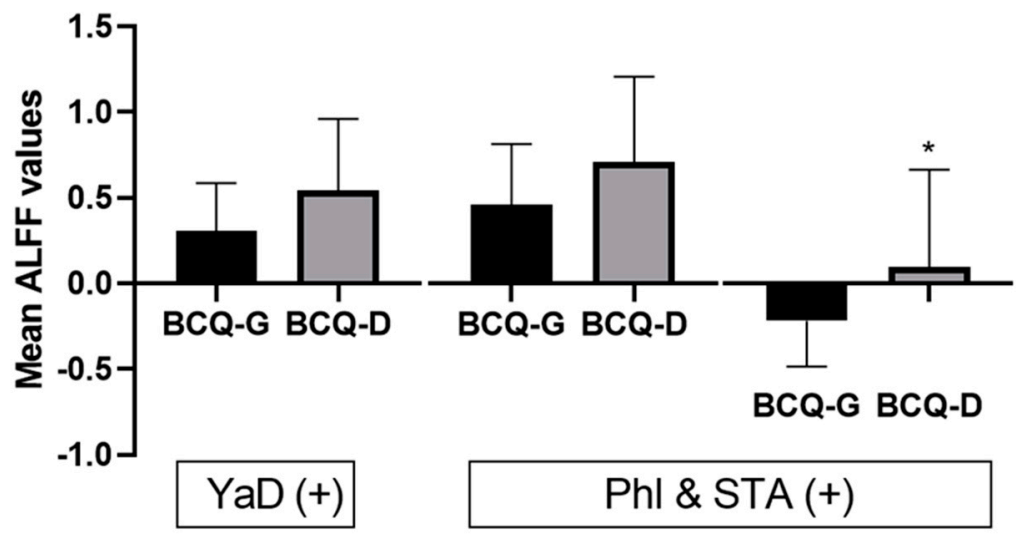

Figure 4. Differences in ALFF values between the groups. The mean ALFF values extracted from the related brain regions (Parahippo1 [BA 36], orbitofrontal cortex, and Parahippo2 [BA 36], which were positively correlated to YaD and Phl \& STA, respectively) nevertheless, showed increased values than those in the BCQ-D group, and significant differences in the region of Parahippo2 between BCQ-G and BCQ-D groups. * $p<0.05$; ReHo, regional homogeneity; ALFF, amplitude of low-frequency fluctuation; $\mathrm{BCQ}$, body constitution questionnaire; SMA, supplementary motor area; Parahippo, parahippocampus; BA, Brodmann area; YinD, yin-deficiency; YaD, yang-deficiency; Phl \& STA, phlegm and stasis.

\section{Discussion}

To the best of our knowledge, this is the first study to use rsfMRI to evaluate BC according to TCM theory. We have explored the changes in spontaneous brain activity in healthy adults with two types of BC, BCQ-G, and BCQ-D. Compared with the BCQ-G group, the BCQ-D group had decreased ReHo values in the right postcentral gyrus (associated with the primary somatosensory cortex). Further, the BCQ-D group had increased ALFF values in the left pons/cerebellum.

Decreased ReHo in the right somatosensory cortex of BCQ-D individuals was associated with WHOQOL-BREF scores for better physiological functions, which meant an adaptive alternation of 
ReHo in these individuals. However, increased ALFF in the brainstem of BCQ-D individuals was associated with lower total WHOQOL-BREF scores, which meant a maladaptive alteration of ALFF in these individuals. In addition, the BCQ subtype analysis $(n=32)$ found that increased ALFF in the parahippocampus and OFC was associated with more points in $\mathrm{YaD}$ and $\mathrm{Phl} \& \mathrm{STA}$ subtypes. Nevertheless, there was a nonsignificant correlation between ReHo and BCQ-subtype. Our results suggested ReHo values in the primary somatosensory cortex may contribute to the homeostasis in both BCQ type and increased ALFF in the parahippocampus may play a role in deficiency-type of BC. Besides, ALFF analysis may exhibit an attenuation of neural activity but may not be seen in ReHo.

The major findings of this study show that BCQ-D individuals had significantly decreased ReHo values in the postcentral gyrus and increased ALFF values in the pons/cerebellum (peduncle). The postcentral gyrus corresponds to the primary somatosensory cortex (BA 3, 1,2), which is associated with conscious perception of pain (location, intensity, and noxious stimulation), somatic sensation, and proprioception $[30,31]$. The primary somatosensory cortex plays a significant role in processing afferent somatosensory input and contributes to integrating the sensory and motor signals for necessary movement [32]. The sub-items of BCQ questionnaire about YinD including the hot sensation over chest/palm/sole area, tinnitus, and dry mouths and lips; and YaD including the sudden blackout in vision, cold intolerance/cold limbs and weakness/coldness/ache of waist, knee and heels and vertigo $[33,34]$. Therefore, comparing to BCQ-G group, the BCQ-D exhibited decreased ReHo in the postcentral gyrus which may pertain to the alteration in the primary sensory modalities involving body sensation (e.g., vestibular and somatosensation) and from external environment stimulation (e.g., hearing and vision) [32,35].

Previous fMRI studies on the functional disorder or pre-disease state have reported increased ReHo activity in the postcentral gyrus associated with irritable bowel syndrome (IBS) with depression and mild cognitive impairment (MCI) respectively $[36,37]$. Moreover, experimentally induced low back pain in healthy subjects showed decreased ReHo in the primary somatosensory cortex after painful stimulation [38]. Therefore, altered regional homogeneity in the postcentral gyrus may contribute to pain procession and emotional dysfunction of the sensorimotor network. Besides, recent studies revealed that a deficiency in yin and yang is a frequent syndrome pattern in Parkinson disease (PD) and diabetes $[39,40]$. These reports suggest that the deficiency syndrome pattern may correlate with neuronal pathophysiology. Our result of BCQ-D individuals showing decreased ReHo in the postcentral gyrus may imply that BCQ-D group may have potential alterations in multi-networks of pain processing, movement coherence, and systemic metabolic arrangement.

Additionally, the BCQ-D individuals showed significant ALFF increases in the left brainstem(pons)/ cerebellum, which was also reported by previous studies in migraineurs (pons) and patients with social anxiety disorder (cerebellum) [41,42]. Pain transmission is linked to the brainstem and associated with the descending pain modulatory system [43]. The cerebellum is in charge of motor control and coordination; and also engaging in pain and emotional processing [44,45]. However, the association between BCQ-D and potential pain-perception disorder or neuropsychiatric abnormality remains unknown.

The correlation analysis revealed that increased ReHo coherence in the cerebellum (lingual)/lingual gyrus and postcentral gyrus was associated with increased/decreased physiological scores in the WHOQOL-BREF subdomain in the BCQ-D group. The anterior lobe of the cerebellum (lingula) receives vestibular connections from the brainstem, mediates body posture, and may connect to the primary somatomotor cortex [46,47]. Increased ReHo in the right cerebellum is associated with increased visual organizational skills [48]. Additionally, neural coherence decreased in the postcentral gyrus of BCQ-D individuals, which was negatively correlated to the physiological subdomain of the WHOQOL-BREF. These results are consistent with previous findings of ALFF of increasing ReHo in the postcentral gyrus may relate to healthy adults with the functional disorder of IBS with depression or MCI [36,37], and decreased ReHo in the postcentral gyrus may associate with better physiological function in our study of BCQ-D group. This suggests that increased local coherence in the cerebellum/lingual gyrus and decreased ReHo in the postcentral gyrus may contribute to 
functional integration between cerebellar-cortical circuits and somatosensory network to maintain daily physiological activity homeostatically in the BCQ-D group. Besides, increased ALFF values were shown in the brainstem and vmPFC in BCQ-D individuals, which were negatively correlated with total WHOQOL-BREF and social subdomain scores. A previous study assessing lower back pain had shown that increased ALFF in the inferior temporal gyrus was negatively correlated with the activities of daily living of Barthel index for activities of daily living [49], but our results revealed that the brainstem (pons) was a region that reflects QOL. Furthermore, the previous studies linking vmPFC activity to placebo analgesia, fear extinction and generalization which may suggest a general role of vmPFC in inhibiting negative emotion [50,51], yet still have evidence that the vmPFC plays a role in the generation of negative emotion [52-54]. Our current result of decreased ALFF in the vmPFC associated with the better social function provided evidence about the vmPFC related to a negative feeling.

In the present study, we used a novel approach utilizing modern rsfMRI with BC analysis, based on TCM. We sought to assess the association between subtypes of BC with brain endophenotypes. Though there were no survival voxels passed under FWE correction in ReHO analysis, the ALFF analysis of correlation with BCQ subtype showed that ALFF values had a significantly positive association with yang-deficiency in the left parahippocampus, and with Phl \& STA in the left parahippocampus and OFC. In subgroup analysis, the subscore of Ph1 \& STA in BCQ-D group revealed significantly increased ALFF in the parahippocampus than in BCQ-G group. (Figure 4). In TCM theory, YaD constitution was commonly coincident with Phl \& STA. The unbalanced BC may exhibit unbalanced condition between qi, blood, yin and yang, frequently determine an individual's susceptibility to certain pathogenic condition and related diseases [3].

A previous rsfMRI meta-analysis suggested that increased ReHo activity in first-episode drug-naive patients with major depressive disorder (MDD) was predominantly located in the left SMA, left parahippocampal gyrus, and hippocampus [55]. In our study, hyperactivity was found in the left parahippocampus (BA 36), which positively correlated with Phl \& STA subscores in the BCQ-D group. Therefore, increased parahippocampal activity may be a possible risk factor of depressive mood that contributes to BC deficiencies, specifically linked to phlegm and stagnation. In TCM, MDD patients were classified as TCM syndrome of deficiency pattern based on the clinical syndrome, pulse, and lingual sign [56]. MDD syndrome is associated with liver stagnation, heart-spleen deficiency, and phlegm misting the mind [57]. However, the relationship between BCQ-D and prevalence of depression remains unclear. Additionally, there were significant positive correlations between ALFF values in the OFC and the Phl \& STA subgroup (Figure 4). The OFC is engaged in executive function and behavioral processing that can inhibit neural activity associated with contextually irrelevant, unwanted information (for example, painful sensations and negative emotions) [58]. A previous ALFF study has shown that cognitive vulnerability to depression was associated with reduced ALFF values in the bilateral OFC [59]. In our study, neural activity in the OFC was positively correlated with Phl \& STA group scores, indicating that individuals in the BCQ-D group may activate the OFC to compensate for negative information and maintain body-mind homeostasis.

ReHo and ALFF are the majority of analytic techniques for rsfMRI data that address low frequency fluctuations. The former is computed only from the direct neighborhood of single voxels, and the latter calculates the voxel-wise magnitude of specific frequency bands in the frequency domain [21]. ReHo may be more sensitive to regional abnormalities than ALFF. Complementarily, ALFF may measure local spontaneous activity [27]. In the present study, we use the 2 indices of low frequency fluctuations of rsfMRI (ReHo, ALFF) to differ the two kinds of body constitution. Our present results demonstrated that ReHo analysis did not reveal the significant neural-behavior correlation of WHOQOL-BREF/BCQ-subtypes in BCQ-G/BCQ-all groups, respectively, which inferred that ReHo might be more sensitive inter-regional coherence. Nevertheless, ALFF may depict a significant negative association with WHOQOL-BREF/BCQ-subtypes (Tables 4 and 5), which implied that ALFF may detect alteration of local spontaneous activity. 
The current study has several limitations. First, we used a cross-sectional method, and the participants were scanned, and BCQ type was assessed for the first time. It is possible that BCQ type may change in different timings and environments. Second, the relatively small sample size in the present study may have diminished the ability to identify more neural activity changes. Third, there were inconsistent methods for classifying BC (for example, CCMQ and BCQ), which limits the reproducibility of our results. Finally, it is unknown whether the presently observed ReHo/ALFF alterations are associated with any subsequent development of pathological conditions. Longitudinal behavior assessments and neuroimaging studies of individuals with the BCQ-D phenotype are required to elucidate these results.

\section{Conclusions}

In conclusion, our combined ReHo and ALFF analyses revealed a significant decrease and increase in spontaneous brain activity, respectively, in various brain regions before structural changes in healthy adults of BCQ deficiency type. The BC subtype analysis showed that BCQ-G participants had consistent neural coherence in ReHo; however, BCQ-D individuals had attenuated neural activity in ALFF. This aberrant neural activity in the brains of BCQ-D individuals indicated an adaptive response to the QOL of these individuals. Future directions relating the BCQ deficiency type to psychological/physiological abnormalities should investigate the underlying mechanisms associated with changes in spontaneous activity in the resting brain state and recognize the importance of $\mathrm{BC}$ in individualizing the treatment strategies in TCM.

Supplementary Materials: The following are available online at http://www.mdpi.com/2077-0383/8/7/951/s1, Figure S1: The flow chart of the study design, Figure S2: The correlation between ALFF and BCQ-subtypes.

Author Contributions: Conceptualization, F.-P.C., Y.-Y.K., J.-C.H. and T.-C.Y.; methodology, H.-C.L., T.-C.Y., C.-J.Y. and P.-S.H.; software, C.-H.L.; investigation, Y.-Y.K., J.-L.Y., T.-P.W. and C.-M.C.; formal analysis, C.-H.L., C.-J.Y.; data curation, P.-S.H., J.-C.H.; writing—original draft preparation, C.-H.L.; writing—review \& Editing, P.-S.H., F.-P.C. and J.-C.H.; project administration, F.-P.C.; supervision, F.-P.C.

Funding: This work was supported by grants from the Lotung Poh-Ai Hospital (Ilan, Taiwan; grant no. 10605-E235) and Taipei Veterans General Hospital (Taipei, Taiwan; grant no. V107C-058).

Acknowledgments: The authors would like to thank Yi-Chang Su, School of Chinese Medicine and Graduate Institute of Chinese Medicine, China Medical University, Taichung, Taiwan, ROC, for kindly providing the use right of 'body constitution questionnaire' to this study. This work is particularly supported by "Development and Construction Plan" of the School of Medicine, National Yang-Ming University. The authors also thank colleagues from the Integrated Brain Research Unit, Department of Medical Research, Taipei Veterans General Hospital, Taipei, Taiwan, ROC, for providing insight and expertise that greatly assisted the research.

Conflicts of Interest: The authors declare no conflicts of interest.

\section{References}

1. Chan, R.Y.; Chien, W.T. Concepts of body constitution, health and sub-health from traditional Chinese medicine perspective. World J. Transl. Med. 2013, 2, 56-66. [CrossRef]

2. Hong, T.-Z. Challenges in learning and understanding traditional Chinese medicine and acupuncture. Open Acc. J. Complement. Altern. Med. Acupunct. 2018, 1, OAJCAM. [CrossRef]

3. Sun, Y.; Zhao, Y.; An, X.S.; Chen, J. The theory development of traditional Chinese medicine constitution: A review. J. Tradit. Chin. Med. Sci. 2018, 5, 16-28. [CrossRef]

4. Su, Y.C.; Chen, L.L.; Lin, J.D.; Lin, J.S.; Huang, Y.C.; Lai, J.S. BCQ+: A body constitution questionnaire to assess Yang-Xu. Part I: Establishment of a first final version through a Delphi process. Forsch. Komplementarmed. 2008, 15, 327-334.

5. Wang, Q.; Zhu, Y.B.; Xue, H.S.; Li, S. Primary compiling of constitution in Chinese medicine questionnaire. Chin. J. Clin. Rehab. 2006, 10, 12-14.

6. Wang, Q.; Yao, S. Molecular basis for cold-intolerant yang-deficient constitution of traditional Chinese medicine. Am. J. Chin. Med. 2008, 36, 827-834. [CrossRef] [PubMed] 
7. Wang, Q.; Yao, S.L.; Dong, J.; Wu, H.D.; Wu, C.Y.; Xia, Z.Y.; Shi, H.F.; Pang, G.M.; Deng, Q.W.; Zhao, J.X.; et al. Changes of endocrine and immune function in subjects of yang deficiency constitution. J. Chin. Integr. Med. 2008, 6, 1226-1232. [CrossRef]

8. Yu, R.; Liu, D.; Yang, Y.; Han, Y.; Li, L.; Zheng, L.; Wang, J.; Zhang, Y.; Li, Y.; Wang, Q.F.; et al. Expression profiling-based clustering of healthy subjects recapitulates classifications defined by clinical observation in Chinese medicine. J. Genet. Genom. 2017, 44, 191-197. [CrossRef]

9. Wang, R.; Wang, J.; Guo, L.L. Effect of traditional Chinese medicine on coronary heart disease with phlegm and blood stasis syndrome. Chin. J. Chin. Mater. Med. 2016, 41, 35-37.

10. Han, Y.M. ZLZLK: Characteristic of constitution theory in traditional Chinese medicine. Zhongyi Linchuang Kangfu 2005, 9, 130-131.

11. Scognamiglio, C.; Houenou, J. A meta-analysis of fMRI studies in healthy relatives of patients with schizophrenia. Aust. N. Z. J. Psychiatry 2014, 48, 907-916. [CrossRef] [PubMed]

12. Webb, C.A.; Dillon, D.G.; Pechtel, P.; Goer, F.K. Neural correlates of three promising endophenotypes of depression: Evidence from the EMBARC study. Neuropsychopharmacology 2016, 41, 454-463. [CrossRef] [PubMed]

13. Ogawa, S.; Lee, T.M.; Kay, A.R.; Tank, D.W. Brain magnetic resonance imaging with contrast dependent on blood oxygenation. Proc. Natl. Acad. Sci. USA 1990, 87, 9868-9872. [CrossRef] [PubMed]

14. Biswal, B.; Yetkin, F.Z.; Haughton, V.M.; Hyde, J.S. Functional connectivity in the motor cortex of resting human brain using echo-planar MRI. Magn. Reson. Med. 1995, 34, 537-541. [CrossRef] [PubMed]

15. Smith, K. Brain imaging: fMRI 2.0. Nature 2012, 484, 24-26. [CrossRef] [PubMed]

16. Guerra-Carrillo, B.; Mackey, A.P.; Bunge, S.A. Resting-state fMRI: A window into human brain plasticity. Neuroscientist 2014, 20, 522-533. [CrossRef] [PubMed]

17. He, T.; Zhu, W.; Du, S.Q.; Yang, J.W.; Li, F.; Yang, B.F.; Shi, G.X.; Liu, C.Z. Neural mechanisms of acupuncture as revealed by fMRI studies. Auton. Neurosci. 2015, 190, 1-9. [CrossRef] [PubMed]

18. Cai, R.L.; Shen, G.M.; Wang, H.; Guan, Y.Y. Brain functional connectivity network studies of acupuncture: A systematic review on resting-state fMRI. J. Integr. Med. 2018, 16, 26-33. [CrossRef]

19. Lin, C.; Zhou, Z.; Xu, J.; Li, Q.; Guo, J.; Long, M.; Wu, D.; Zhang, Y. Changes of brain activity during a functional magnetic resonance imaging stroop task study: Effect of Chinese herbal formula in Alzheimer's disease. Eur. J. Integr. Med. 2017, 16, 46-53. [CrossRef]

20. Xu, Z.; Zhang, S.; Huang, L.; Zhu, X.; Zhao, Q.; Zeng, Y.; Zhou, D.; Wang, D.; Kuga, H.; Kamiya, A.; et al. Altered resting-state brain activities in drug-naive major depressive disorder assessed by fMRI: Associations with somatic symptoms defined by Yin-Yang theory of the traditional Chinese medicine. Front. Psychiaty 2018, 9, 195. [CrossRef]

21. Margulies, D.S.; Bottger, J.; Long, X.; Lv, Y.; Kelly, C.; Schafer, A.; Goldhahn, D.; Abbushi, A.; Milham, M.P.; Lohmann, G.; et al. Resting developments: A review of fMRI post-processing methodologies for spontaneous brain activity. Magma 2010, 23, 289-307. [CrossRef] [PubMed]

22. Lv, H.; Wang, Z. Resting-state functional MRI: Everything that nonexperts have always wanted to know. Am. J. Neuroradiol. 2018, 39, 1390-1399. [CrossRef] [PubMed]

23. Zang, Y.; Jiang, T.; Lu, Y.; He, Y.; Tian, L. Regional homogeneity approach to fMRI data analysis. Neuroimage 2004, 22, 394-400. [CrossRef] [PubMed]

24. Zang, Y.F.; He, Y.; Zhu, C.Z.; Cao, Q.J.; Sui, M.Q.; Liang, M.; Tian, L.X.; Jiang, T.Z.; Wang, Y.F. Altered baseline brain activity in children with ADHD revealed by resting-state functional MRI. Brain Dev. 2007, 29, 83-91. [PubMed]

25. Cui, Y.; Jiao, Y.; Chen, Y.C.; Wang, K.; Gao, B.; Wen, S.; Ju, S.; Teng, G.J. Altered spontaneous brain activity in type 2 diabetes: A resting-state functional MRI study. Diabetes 2014, 63, 749-760. [CrossRef] [PubMed]

26. Xu, Y.; Zhuo, C.; Qin, W.; Zhu, J.; Yu, C. Altered spontaneous brain activity in schizophrenia: A meta-analysis and a large-sample study. BioMed Res. Int. 2015, 2015, 204628. [CrossRef] [PubMed]

27. An, L.; Cao, Q.J.; Sui, M.Q.; Sun, L.; Zou, Q.H.; Zang, Y.F.; Wang, Y.F. Local synchronization and amplitude of the fluctuation of spontaneous brain activity in attention-deficit/hyperactivity disorder: A resting-state fMRI study. Neurosci. Bull. 2013, 29, 603-613. [CrossRef] [PubMed]

28. Yao, G.; Wu, C.H. Factorial invariance of the WHOQOL-BREF among disease groups. Qual. Life Res. 2005, 14, 1881-1888. [CrossRef] [PubMed] 
29. Yan, C.G.; Wang, X.D.; Zuo, X.N.; Zang, Y.F. DPABI: Data processing \& analysis for (resting-state) brain imaging. Neuroinformatics 2016, 14, 339-351.

30. Kanda, M.; Nagamine, T.; Ikeda, A.; Ohara, S.; Kunieda, T.; Fujiwara, N.; Yazawa, S.; Sawamoto, N.; Matsumoto, R.; Taki, W.; et al. Primary somatosensory cortex is actively involved in pain processing in human. Brain Res. 2000, 853, 282-289. [CrossRef]

31. Baehr, M.F.M. Duus' Topical Diagnosis in Neurology: Anatomy, Physiology, Signs, Symptoms, 4th ed.; Thieme: New York, NY, USA, 2005.

32. Borich, M.R.; Brodie, S.M.; Gray, W.A.; Ionta, S.; Boyd, L.A. Understanding the role of the primary somatosensory cortex: Opportunities for rehabilitation. Neuropsychologia 2015, 79, 246-255. [CrossRef] [PubMed]

33. Chen, L.L.; Lin, J.S.; Lin, J.D.; Chang, C.H.; Kuo, H.W.; Liang, W.M.; Su, Y.C. BCQ+: A body constitution questionnaire to assess Yang-Xu. Part II: Evaluation of reliability and validity. Forsch. Komplementarmed. 2009, 16, 20-27.

34. Lin, J.S.; Chen, L.L.; Lin, J.D.; Chang, C.H.; Huang, C.H.; Mayer, P.K.; Su, Y.C. BCQ-: A body constitution questionnaire to assess Yin-Xu. Part II: Evaluation of reliability and validity. Forsch. Komplementarmed. 2012, 19, 285-292. [CrossRef] [PubMed]

35. Riemann, B.L.; Lephart, S.M. The sensorimotor system, Part II: The role of proprioception in motor control and functional joint stability. J. Athlet. Train. 2002, 37, 80-84.

36. Li, J.; Li, G.X.; Guo, Y.; Lu, X.Q.; Li, L.; Ding, J.P. Regional homogeneity in the patients of irritable bowel syndrome complicated with depression: A resting-state functional magnetic resonance imaging study. Zhonghua Yi Xue Za Zhi 2018, 98, 196-201. [PubMed]

37. Zhen, D.; Xia, W.; Yi, Z.Q.; Zhao, P.W.; Zhong, J.G.; Shi, H.C.; Li, H.L.; Dai, Z.Y.; Pan, P.L. Alterations of brain local functional connectivity in amnestic mild cognitive impairment. Transl. Neurodegen. 2018, 7, 26. [CrossRef] [PubMed]

38. Zhang, S.S.; Wu, W.; Liu, Z.P.; Huang, G.Z.; Guo, S.G.; Yang, J.M. Altered regional homogeneity in experimentally induced low back pain: A resting-state fMRI study. J. Neuroeng. Rehabil. 2014, 11, 115. [CrossRef]

39. Chen, H.; He, J.; Teng, L.; Yuan, C.; Zhang, Z. Traditional Chinese medicine symptom pattern analysis for Parkinson's disease. J. Tradit. Chin. Med. 2017, 37, 688-694.

40. Guo, J.; Chen, H.; Song, J.; Wang, J.; Zhao, L.; Tong, X. Syndrome differentiation of diabetes by the traditional Chinese medicine according to evidence-based medicine and expert consensus opinion. eCAM 2014, 2014, 492193. [CrossRef]

41. Wang, J.J.; Chen, X.; Sah, S.K.; Zeng, C.; Li, Y.M.; Li, N.; Liu, M.Q.; Du, S.L. Amplitude of low-frequency fluctuation (ALFF) and fractional ALFF in migraine patients: A resting-state functional MRI study. Clin. Radiol. 2016, 71, 558-564. [CrossRef]

42. Yuan, C.; Zhu, H.; Ren, Z.; Yuan, M.; Gao, M.; Zhang, Y.; Li, Y.; Meng, Y.; Gong, Q.; Lui, S.; et al. Precuneus-related regional and network functional deficits in social anxiety disorder: A resting-state functional MRI study. Compr. Psychiatry 2018, 82, 22-29. [CrossRef] [PubMed]

43. Tracey, I.; Mantyh, P. The cerebral signature for pain perception and its modulation. Neuron 2007, 55, $377-391$. [CrossRef]

44. Garcia-Larrea, L.; Peyron, R. Pain matrices and neuropathic pain matrices: A review. Pain 2013, 154, S29-S43. [CrossRef] [PubMed]

45. Schmahmann, J.D.; Weilburg, J.B.; Sherman, J.C. The neuropsychiatry of the cerebellum-Insights from the clinic. Cerebellum 2007, 6, 254-267. [CrossRef] [PubMed]

46. Ito, M. The Cerebellum and Neural Control; Raven Press Books, Ltd.: New York, NY, USA, 1984.

47. Yeo, B.T.; Krienen, F.M.; Sepulcre, J.; Sabuncu, M.R.; Lashkari, D.; Hollinshead, M.; Roffman, J.L.; Smoller, J.W.; Zollei, L.; Polimeni, J.R.; et al. The organization of the human cerebral cortex estimated by intrinsic functional connectivity. J. Neurophysiol. 2011, 106, 1125-1165. [PubMed]

48. Stoodley, C.J.; Valera, E.M.; Schmahmann, J.D. Functional topography of the cerebellum for motor and cognitive tasks: An fMRI study. Neuroimage 2012, 59, 1560-1570. [CrossRef] [PubMed]

49. Zhou, F.; Gu, L.; Hong, S.; Liu, J.; Jiang, J.; Huang, M.; Zhang, Y.; Gong, H. Altered low-frequency oscillation amplitude of resting state-fMRI in patients with discogenic low-back and leg pain. J. Pain Res. 2018, 11, 165-176. [CrossRef] 
50. Atlas, L.Y.; Wager, T.D. A meta-analysis of brain mechanisms of placebo analgesia: Consistent findings and unanswered questions. Handb. Exp. Pharmacol. 2014, 225, 37-69.

51. Hiser, J.; Koenigs, M. The multifaceted role of ventromedial prefrontal cortex in emotion, decision-making, social cognition, and psychopathology. Biol. Psychiatry 2018, 83, 638-647. [CrossRef]

52. Damasio, A.R.; Tranel, D.; Damasio, H. Individuals with sociopathic behavior caused by frontal damage fail to respond autonomically to social stimuli. Behav. Brain Res. 1990, 41, 81-94. [CrossRef]

53. Koenigs, M.; Huey, E.D.; Calamia, M.; Raymont, V.; Tranel, D.; Grafman, J. Distinct regions of prefrontal cortex mediate resistance and vulnerability to depression. J. Neurosci. 2008, 28, 12341-12348. [CrossRef] [PubMed]

54. Koenigs, M.; Huey, E.D.; Raymont, V.; Cheon, B.; Solomon, J.; Wassermann, E.M.; Grafman, J. Focal brain damage protects against post-traumatic stress disorder in combat veterans. Nature Neurosci. 2008, 11, 232-237. [CrossRef] [PubMed]

55. Hao, H.; Chen, C.; Mao, W.; Zhong, J.; Dai, Z. Aberrant brain regional homogeneity in first-episode drug-naive patients with major depressive disorder: A voxel-wise meta-analysis. J. Affect. Disord. 2019, 245, 63-71. [CrossRef] [PubMed]

56. Zhang, Y.F.; Han, Y.; Wang, Y.Z.; Zhang, Y.F.; Jia, H.X.; Jin, E.H.; Deng, L.G.; Li, L. Characterization of resting-state fMRI-derived functional connectivity in patients with deficiency versus excess patterns of major depression. Complement. Ther. Med. 2015, 23, 7-13. [CrossRef] [PubMed]

57. Bosch, P.; de Rover, P.; Staudte, H.; Lim, S.; van den Noort, M. Schizophrenia, depression, and sleep disorders: Their traditional oriental medicine equivalents. J. Acupunct. Meridian Stud. 2015, 8, 17-22. [CrossRef] [PubMed]

58. Shimamura, A.P. The role of the prefrontal cortex in dynamic filtering. Psychobiology 2000, 28, $207-218$.

59. Zhang, X.; Di, X.; Lei, H.; Yang, J.; Xiao, J.; Wang, X.; Yao, S.; Rao, H. Imbalanced spontaneous brain activity in orbitofrontal-insular circuits in individuals with cognitive vulnerability to depression. J. Affect. Disord. 2016, 198, 56-63. [CrossRef] [PubMed]

(C) 2019 by the authors. Licensee MDPI, Basel, Switzerland. This article is an open access article distributed under the terms and conditions of the Creative Commons Attribution (CC BY) license (http://creativecommons.org/licenses/by/4.0/). 